\title{
Effects of lateral position and open chest on lung and chest wall resistance during thoracic surgery
}

\section{Umbrello', P Formenti', G Mistraletti', F Vetrone1, A Marino', G Vergani',}

A Baisi'2, D Chiumello1

UNIVERSITÀ

DEGLI STUDI (1) Anesthesia and Intensive Care, Ospedale San Paolo, Università degli Studi di Milano, Italy Di Milano

(2) Thoracic Surgery, Ospedale San Paolo, Università degli Studi di Milano, Italy

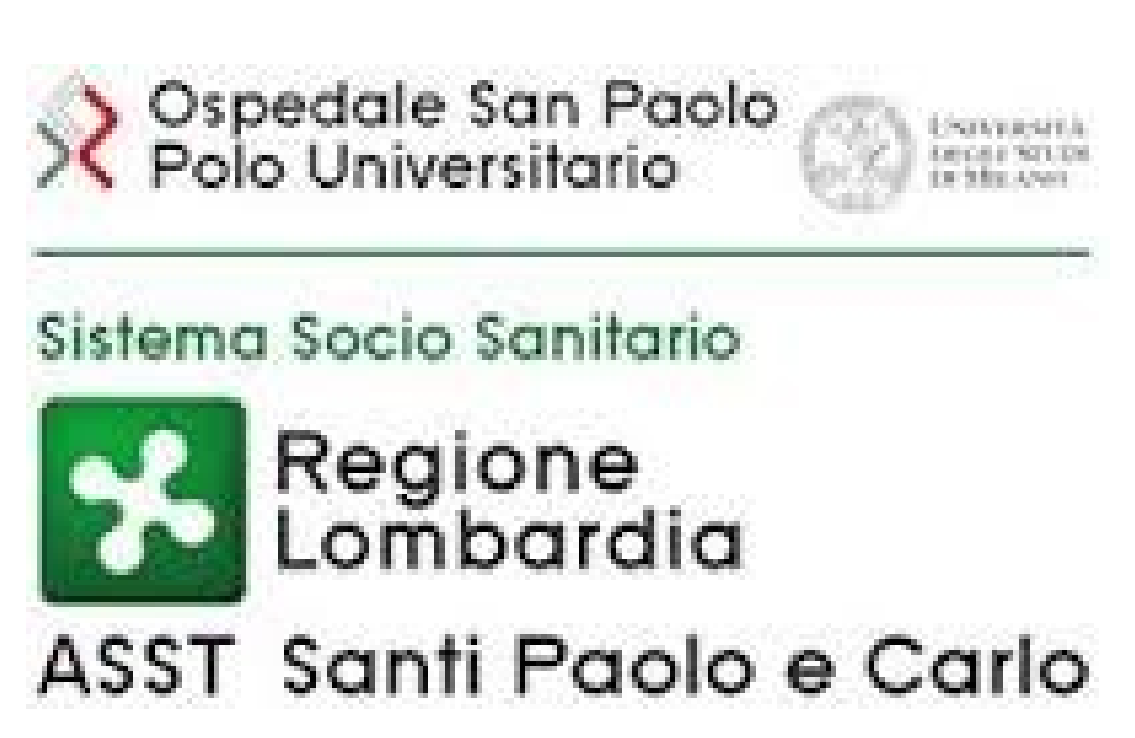

\section{Introduction}

Airway-pressure (Paw) based respiratory mechanics guides mechanical ventilation during anesthesia.

However, altered properties of the chest wall may significantly influence the interpretation of Paw, especially in settings in which an alteration of chest physiology occurs.

Aim of the present study was to investigate the resistive behavior of lung and chest wall during different phases of thoracic surgery.

\section{Methods}

Subjects undergoing thoracotomic pulmonary resection were enrolled.

Double-lung ventilation (DLV): tidal volume (TV) $8 \mathrm{ml} / \mathrm{kg}$, PEEP $8 \mathrm{cmH} 2 \mathrm{O}$, $\mathrm{FiO} 2$ and respiratory rate to maintain $\mathrm{SaO} 294-98 \%$ and $\mathrm{EtCO} 230-35 \mathrm{mmHg}$.

One-lung ventilation (OLV): TV $5 \mathrm{ml} / \mathrm{kg}$ and respiratory rate increased accordingly. Esophageal pressure as a surrogate for pleural pressure.

Maximal respiratory system resistance (Rmax,rs): difference of peak and plateau airway pressure divided by inspiratory flow. Rmax, rs includes flow resistance of airways (Rmin,rs) and that caused by stress relaxation/time

constant inequalities within the tissues (DR,rs). Each resistance was partitioned into their respective lung/chest wall component (Rmax,I; Rmin,l; $\mathrm{DR}, \mathrm{l} ; \mathrm{Rmax}, \mathrm{cw})$. See figure 1

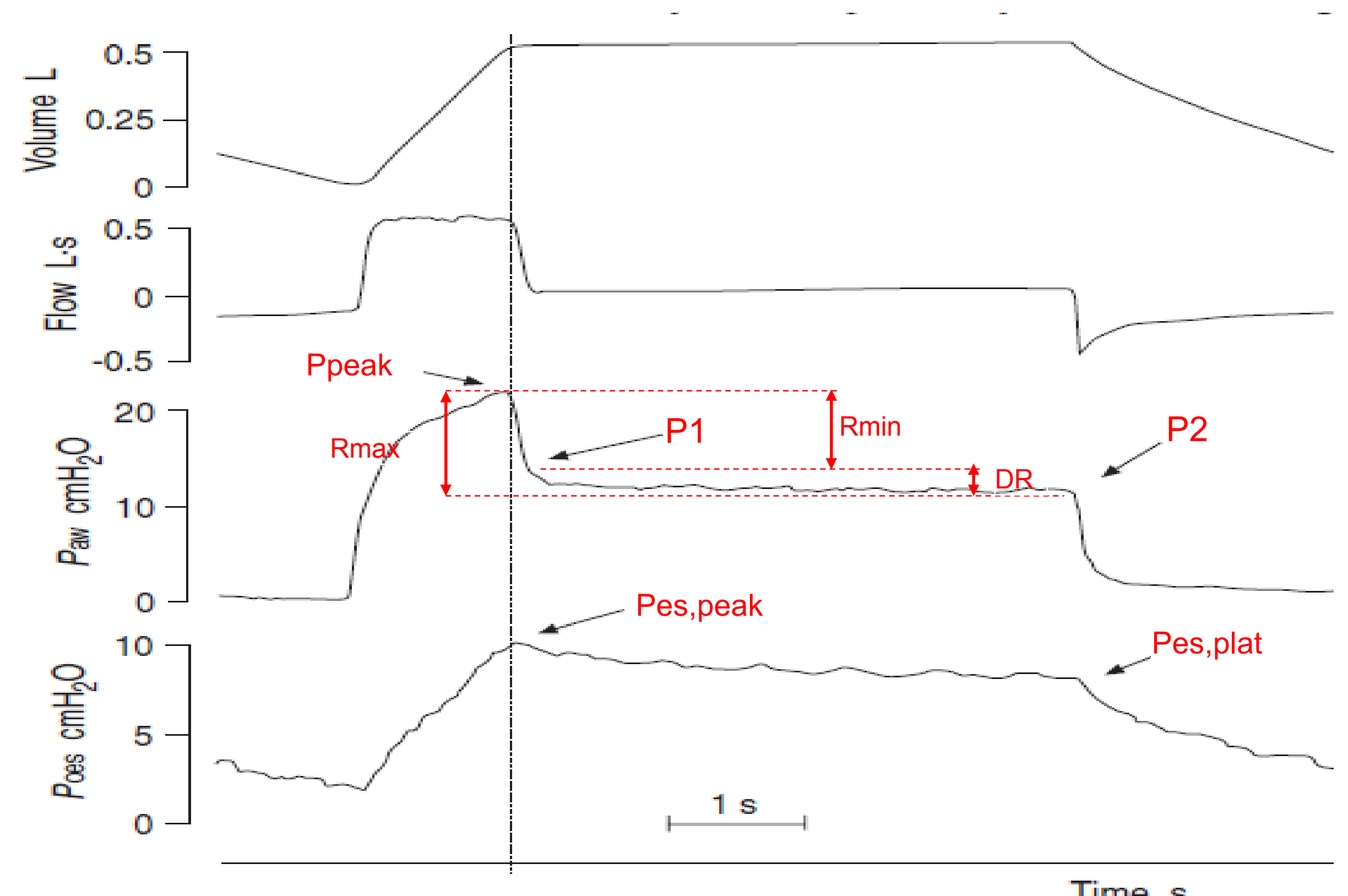

\section{Figure \#1}

Measurements were repeated during DLV in supine and lateral decubitus, OLV in lateral decubitus during closed and open chest conditions (figure 2)

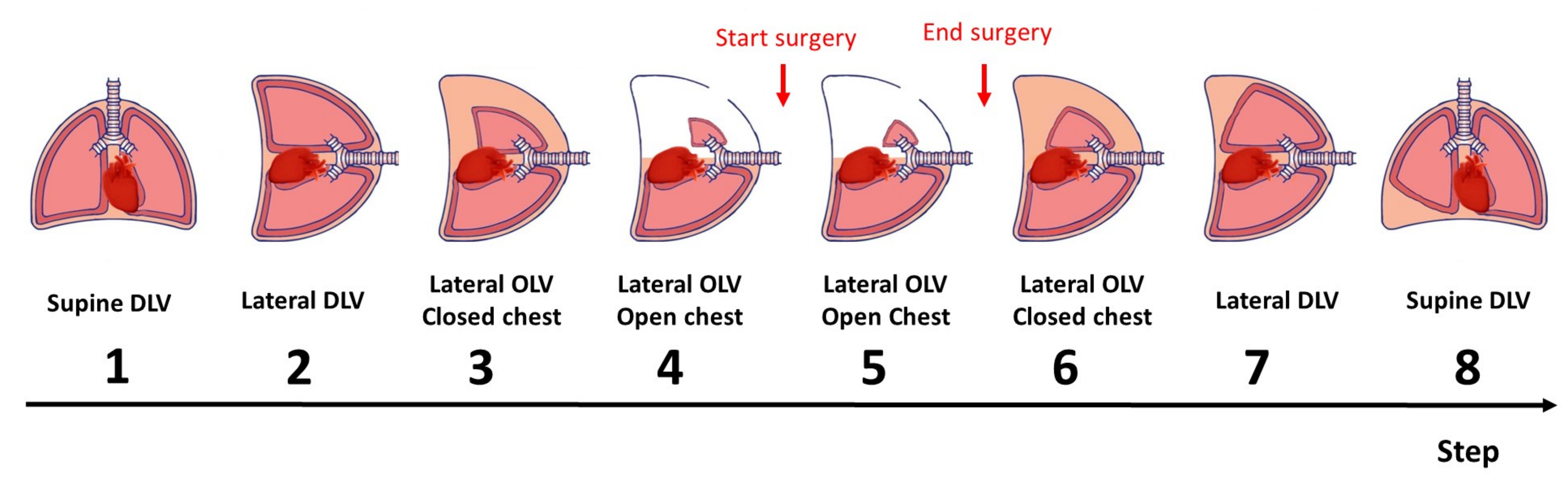

\section{Results}

30 patients were enrolled,

18 males (60\%), age $68 \pm 10$ years.

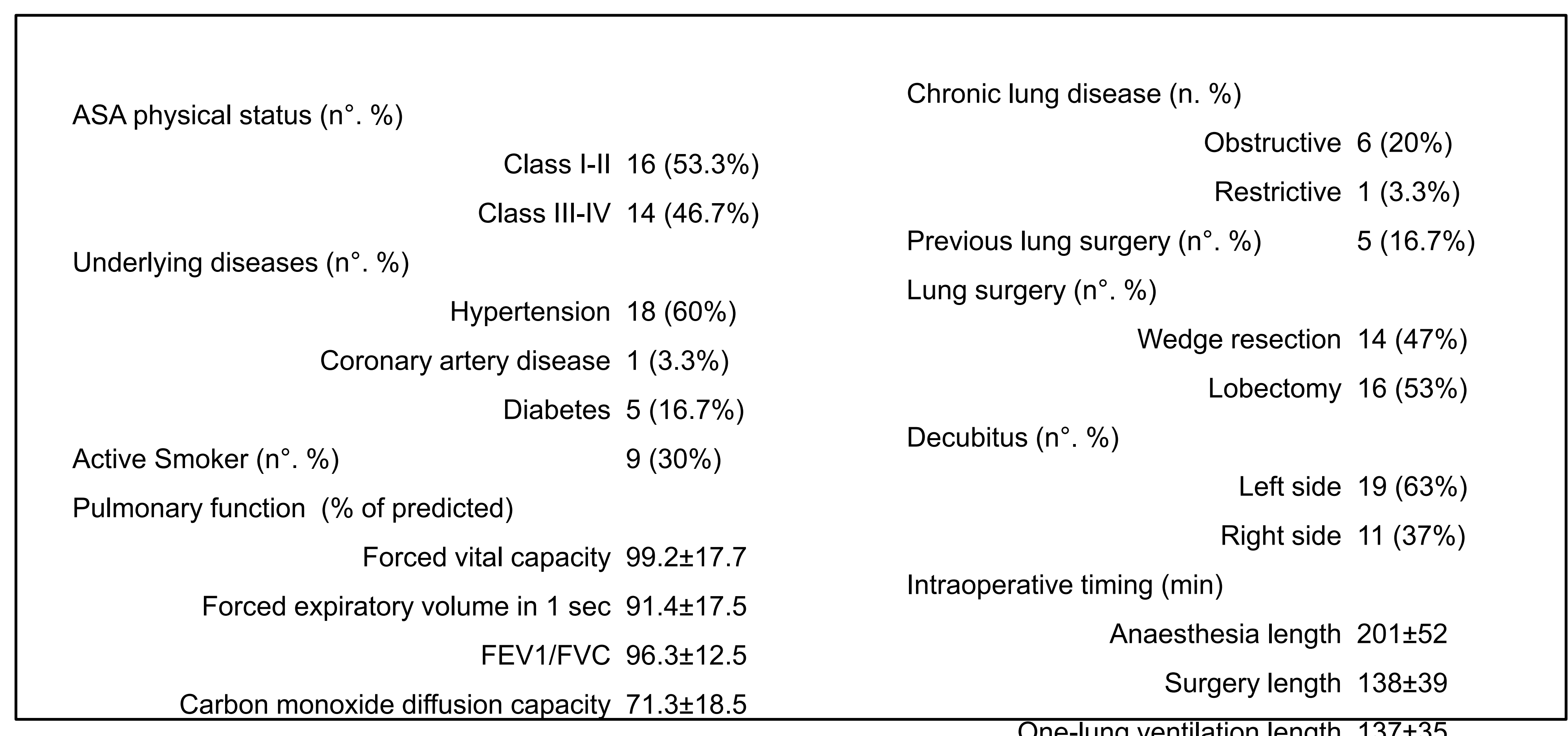

Figure 3 shows the respiratory system, lung and chest wall resistances partitioned into their Ohmic and viscoelastic components during the different study steps

Figure \#3

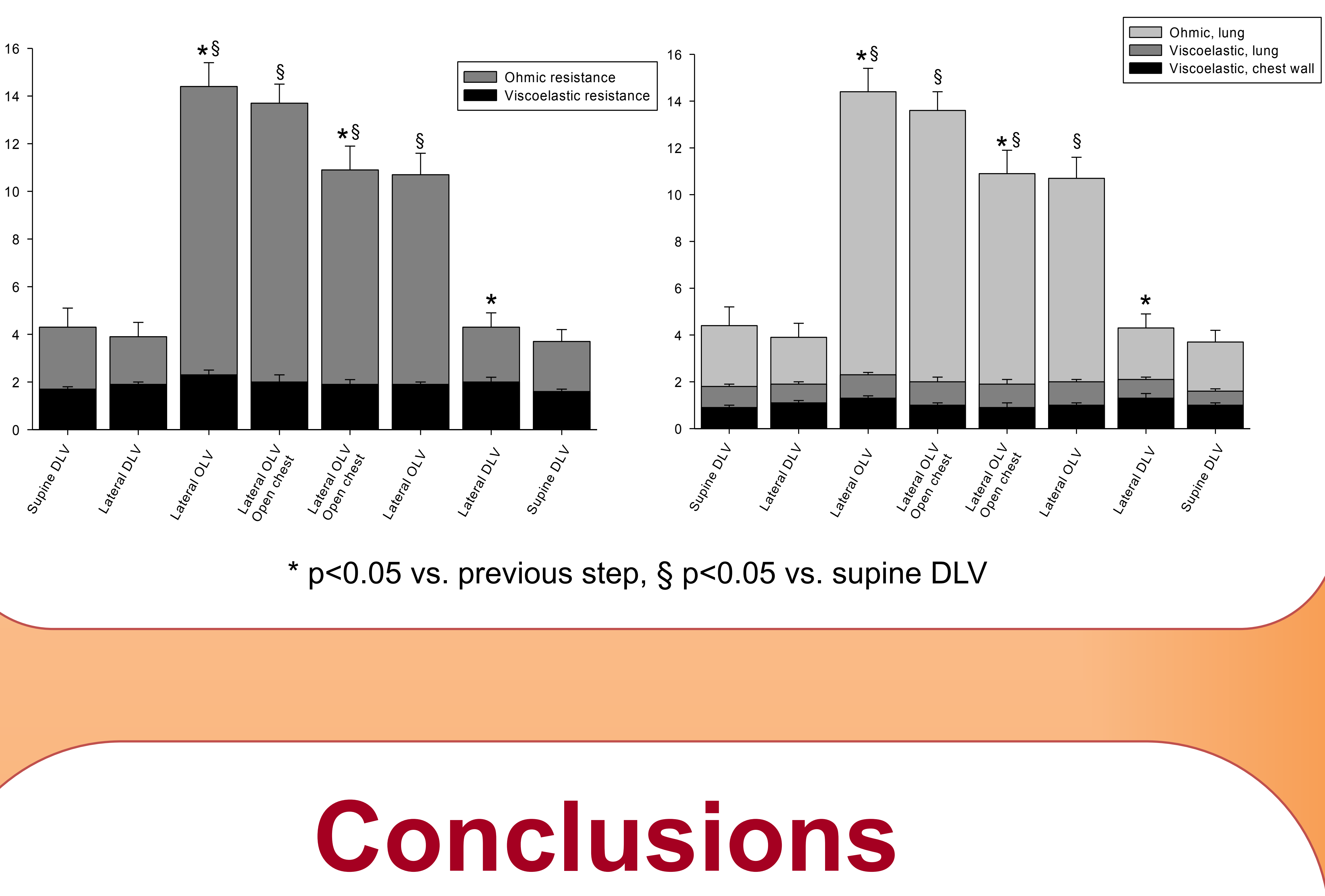

Unexpected decrease in Rmax, rs after lung resection: Possible increase in the radial traction acting on the airways after lung re-expansion, increasing their diameter, then reducing resistance.

A similar phenomenon was seen in lung volume reduction surgery: parenchymal resection brings to an increased slope of the recoil pressure - maximum flow relationship (greater flow at the same pressure), then leading to increased airway conductance

Paw-based monitoring of resistance during the different phases of thoracic surgery does not allow to differentiate the cause of an alteration. In this context, we suggest that partitioning of respiratory mechanics may give a deeper insight into the resistive behavior of the lung and the chest wall.

\section{References}

1. Pelosi, Anesth Analg 1995

2. Barnas, J Cardiothorac Vasc Anesth 1997

3. Gelb, Am J Respir Crit Care Med. 1996 